TRI-PP-97-09, MKPH-T-97-12

\title{
Generalized polarizabilities and the chiral structure of the nucleon
}

\author{
Thomas R. Hemmert ${ }^{1}$, Barry R. Holstein ${ }^{2}$, Germar Knöchlein ${ }^{1,3}$ and Stefan Scherer ${ }^{3}$ \\ 1 TRIUMF, Theory Group, 4004 Wesbrook Mall, Vancouver B. C., Canada V6T $2 A 3$ \\ 2 Department of Physics and Astronomy, University of Massachusetts, Amherst MA 01003, USA \\ ${ }^{3}$ Institut für Kernphysik, Johannes Gutenberg-Universität, D-55099 Mainz, Germany
}

\begin{abstract}
We present results of the first chiral perturbation theory calculation for the generalized polarizabilities of the nucleon and discuss the response functions in virtual Compton scattering to be measured in the scheduled electron scattering experiments.
\end{abstract}

PACS numbers: 11.30.Rd, 12.39.Fe, 13.60.Fz, 14.20.Dh

Typeset using REVTEX 
Compton scattering off the nucleon has a long history as a tool for obtaining precision information on the internal structure of the nucleon. Beyond the low-energy theorem [1] the amplitude for unpolarized Compton scattering of real photons can be described in terms of two structure-dependent coefficients [2], commonly denoted as the electric and magnetic polarizabilities $\bar{\alpha}_{E}$ and $\bar{\beta}_{M}$. Whereas the constituent quark model traditionally has some problems [3] in explaining the numerical values for $\bar{\alpha}_{E}$ and $\bar{\beta}_{M}$, it has been shown that these features can be satisfactorily understood in chiral perturbation theory (ChPT [4,5]), suggesting that $\bar{\alpha}_{E}$ and $\bar{\beta}_{M}$ are dominated by the pion cloud contribution of the nucleon [5.66].

Recently a new frontier in Compton scattering has been defined (see, e.g., [7]) and is now in the beginning of being explored: It has been proposed [8] to study the electron scattering process $e p \rightarrow e^{\prime} p^{\prime} \gamma$ in order to obtain information on the genuine virtual Compton scattering (VCS) process $\gamma^{*} N \rightarrow \gamma N$. In addition to the two kinematical variables of real Compton scattering, e.g. the scattering angle $\theta$ and the energy $\omega^{\prime}$ of the outgoing photon, the invariant amplitude for VCS [9 12] depends on a third kinematical variable, where we will use the absolute value of the three-momentum transfer to the nucleon in the hadronic c.m. frame, $\bar{q} \equiv|\vec{q}|$. The structure-dependent coefficients in the VCS amplitude as defined in [11] acquire a $\bar{q}$ dependence and are termed "generalized polarizabilities" (GPs) of the nucleon in analogy to the structure coefficients in real Compton scattering. However, due to the specific kinematic approximation chosen there is no one-to-one correspondence between all the real Compton polarizabilities and the GPs of Guichon et al. in VCS [11, 13, 14].

The virtual nature of the initial state photon with the associated possibility of an independent variation of photon energy and momentum allows for scanning the momentum dependence of the GPs in the electron scattering experiment, thus rendering access to a much greater variety of structure information than in the case of real Compton scattering. In particular, one hopes to identify the individual signatures of specific nucleon resonances in the various GPs, which cannot be obtained in other processes [7]. In this letter, we will only discuss VCS below the pion-production threshold. For an overview of the status at 
higher energies and in the deep inelastic regime we refer to [7].

So far, predictions for both types of GPs - spin-independent and spin-dependent-have been available within a non-relativistic constituent quark model [11] and a one-loop calculation in the linear sigma model [15]. Various approaches have been used to calculate the two spin-independent polarizabilities $\bar{\alpha}_{E}\left(\bar{q}^{2}\right)$ and $\bar{\beta}_{M}\left(\bar{q}^{2}\right)$, namely, an effective Lagrangian approach including nucleon resonance effects [16], a calculation of the leading $\bar{q}^{2}$ dependence in heavy-baryon ChPT (HBChPT) [17 and a calculation of $\bar{\alpha}_{E}\left(\bar{q}^{2}\right)$ in the Skyrme model [18]. In [13, 14] it was shown that the number of independent GPs is reduced, once charge-conjugation symmetry is imposed.

In this letter we provide the complete set of predictions for the GPs and the measurable response functions using HBChPT. The calculation is performed to third order in the momentum expansion, which is known to work well in the case of real Compton scattering [5.

The GPs of the nucleon have been defined in terms of electromagnetic multipoles as functions of the initial photon momentum $\bar{q}$ [11] ,

$$
\begin{aligned}
P^{\left(\rho^{\prime} L^{\prime}, \rho L\right) S}\left(\bar{q}^{2}\right) & =\left[\frac{1}{\omega^{\prime L} \bar{q}^{L}} H^{\left(\rho^{\prime} L^{\prime}, \rho L\right) S}\left(\omega^{\prime}, \bar{q}\right)\right]_{\omega^{\prime}=0}, \\
\hat{P}^{\left(\rho^{\prime} L^{\prime}, L\right) S}\left(\bar{q}^{2}\right) & =\left[\frac{1}{\omega^{\prime L} \bar{q}^{L+1}} \hat{H}^{\left(\rho^{\prime} L^{\prime}, L\right) S}\left(\omega^{\prime}, \bar{q}\right)\right]_{\omega^{\prime}=0},
\end{aligned}
$$

where $L\left(L^{\prime}\right)$ denotes the initial (final) photon orbital angular momentum, $\rho\left(\rho^{\prime}\right)$ the type of multipole transition $(0=C$ (scalar, Coulomb), $1=M$ (magnetic), $2=E$ (electric) $)$, and $S$ distinguishes between non-spin-flip $(S=0)$ and spin-flip $(S=1)$ transitions. Mixed-type polarizabilities, $\hat{P}^{\left(\rho^{\prime} L^{\prime}, L\right) S}\left(\bar{q}^{2}\right)$, have been introduced, which are neither purely electric nor purely Coulomb type. It is important to note that the above definitions are based on the kinematical approximation that the multipoles are expanded around $\omega^{\prime}=0$ and only terms linear in $\omega^{\prime}$ are retained, which together with current conservation yields selection rules for the possible combinations of quantum numbers of the GPs. In this approximation, 10 GPs have been introduced in [1] as functions of $\bar{q}^{2}: P^{(01,01) 0}, P^{(11,11) 0}, P^{(01,01) 1}, P^{(11,11) 1}$, $P^{(01,12) 1}, P^{(11,02) 1}, P^{(11,00) 1}, \hat{P}^{(01,1) 0}, \hat{P}^{(01,1) 1}, \hat{P}^{(11,2) 1}$. 
However, recently it has been proved [13, 14 that only six of the above ten GPs are independent, if one requires charge-conjugation symmetry to hold. With $\omega_{0} \equiv M-\sqrt{M^{2}+\bar{q}^{2}}$, $M$ being the nucleon mass, the four constraints implied by $C$ invariance can be written as

$$
\begin{aligned}
& 0=\sqrt{\frac{3}{2}} P^{(01,01) 0}\left(\bar{q}^{2}\right)+\sqrt{\frac{3}{8}} P^{(11,11) 0}\left(\bar{q}^{2}\right)+\frac{3 \bar{q}^{2}}{2 \omega_{0}} \hat{P}^{(01,1) 0}\left(\bar{q}^{2}\right), \\
& 0=P^{(11,11) 1}\left(\bar{q}^{2}\right)+\sqrt{\frac{3}{2}} \omega_{0} P^{(11,02) 1}\left(\bar{q}^{2}\right)+\sqrt{\frac{5}{2}} \bar{q}^{2} \hat{P}^{(11,2) 1}\left(\bar{q}^{2}\right), \\
& 0=2 \omega_{0} P^{(01,01) 1}\left(\bar{q}^{2}\right)+2 \frac{\bar{q}^{2}}{\omega_{0}} P^{(11,11) 1}\left(\bar{q}^{2}\right)-\sqrt{2} \bar{q}^{2} P^{(01,12) 1}\left(\bar{q}^{2}\right)+\sqrt{6} \bar{q}^{2} \hat{P}^{(01,1) 1}\left(\bar{q}^{2}\right), \\
& 0=3 \frac{\bar{q}^{2}}{\omega_{0}} P^{(01,01) 1}\left(\bar{q}^{2}\right)-\sqrt{3} P^{(11,00) 1}\left(\bar{q}^{2}\right)-\sqrt{\frac{3}{2}} \bar{q}^{2} P^{(11,02) 1}\left(\bar{q}^{2}\right) .
\end{aligned}
$$

In the scalar (i.e. spin-independent) sector it is convenient to use Eq.(2a) to eliminate the mixed polarizability $\hat{P}^{(01,1) 0}$ in favor of $P^{(01,01) 0}$ and $P^{(11,11) 1}$, because the latter are generalizations of the electric and magnetic polarizabilities in real Compton scattering:

$$
\begin{aligned}
& \bar{\alpha}_{E}\left(\bar{q}^{2}\right)=-\frac{e^{2}}{4 \pi} \sqrt{\frac{3}{2}} P^{(01,01) 0}\left(\bar{q}^{2}\right), \\
& \bar{\beta}_{M}\left(\bar{q}^{2}\right)=-\frac{e^{2}}{4 \pi} \sqrt{\frac{3}{8}} P^{(11,11) 0}\left(\bar{q}^{2}\right) .
\end{aligned}
$$

However, in the spin-dependent sector it is not a priori clear which three GPs should be eliminated with the help of the constraints-Eqs. (2b)-(2d $)$. Thus, and for easier comparison with other theoretical calculations which have been performed before the constraints from Eq. (2) were recognized, we will give results for the original set of 10 GPs. Eqs. (2a) - 2d) then provide a useful check for any calculation of the GPs.

Our calculation of the GPs is performed within HBChPT [5] to third order in the external momenta - $O\left(p^{3}\right)$. The chiral results are highly constrained, the only parameters being the pion mass $m_{\pi}$, the axial coupling constant $g_{A}$, and the pion decay constant $F_{\pi}$, which are all known very accurately. At $O\left(p^{3}\right)$, contributions to the GPs are generated by nine one-loop diagrams and the $\pi^{0}$-exchange $t$-channel pole graph which are displayed in [17]. For the loop diagrams only the leading-order Lagrangians are required [5]:

$$
\begin{aligned}
\mathcal{L}_{\pi N}^{(1)} & =\bar{N}_{v}\left(i v \cdot D+g_{A} S \cdot u\right) N_{v}, \\
\mathcal{L}_{\pi \pi}^{(2)} & =\frac{F_{\pi}^{2}}{4} \operatorname{tr}\left[\left(\nabla_{\mu} U\right)^{\dagger} \nabla^{\mu} U\right] .
\end{aligned}
$$


In the above $S U(2)$ Lagrangians, $N_{v}$ represents a non-relativistic nucleon field, and $U=$ $\exp \left(i \vec{\tau} \cdot \vec{\pi} / F_{\pi}\right)$ contains the pion field. Standard covariant derivatives have been introduced acting on the pion $\left(\nabla_{\mu} U\right)$ and on the nucleon $\left(D_{\mu} N_{v}\right)$, in addition to the chiral vielbein $u_{\mu}$, which contains the derivative coupling of a pion. In the heavy-baryon Lagrangian one also must specify the velocity vector $v_{\mu}$, which determines the representation of the PauliLubanski spin-vector $S^{\mu}=i \gamma_{5} \sigma^{\mu \nu} v_{\nu}$ [5]. Finally, for the $\pi^{0}$-exchange diagram we require in addition to Eq. (四) the anomalous $\pi^{0} \gamma \gamma^{*}$ vertex provided by the Wess-Zumino-Witten Lagrangian [19],

$$
\mathcal{L}_{\gamma \gamma \pi^{0}}^{(W Z W)}=-\frac{e^{2}}{32 \pi^{2} F_{\pi}} \epsilon^{\mu \nu \alpha \beta} F_{\mu \nu} F_{\alpha \beta} \pi^{0},
$$

where $\epsilon_{0123}=1$ and $F_{\mu \nu}$ is the electromagnetic field strength tensor. Details of the calculation will be reported in a separate publication.

In [17] we have already performed a calculation of the first derivative of the spinindependent polarizabilities with respect to $\bar{q}^{2}$, and technically the calculation of the material presented here proceeds along the same line. However, [17 focuses on the VCS regime of very low momentum transfer $\left(\omega^{\prime}, \bar{q} \ll m_{\pi}\right)$, for which the formalism introduced in [11] is not applicable. The extraction of the GPs from a heavy-baryon calculation is described in detail in Sec. IV of [14]. The numerical results for the ten generalized proton polarizabilities are presented in Fig.1. Therein, the contribution from the anomalous Wess-Zumino-Witten interaction only arises in the spin-dependent sector and is plotted separately.

Let us first discuss the electric polarizability $\bar{\alpha}_{E}\left(\bar{q}^{2}\right)$, which is numerically by far the largest of the ten GPs and thus can presumably be determined most reliably in the scheduled experiments. At the kinematic point $\bar{q}^{2}=0$ the generalized electric polarizability $\bar{\alpha}_{E}\left(\bar{q}^{2}\right)$ can be identified with the electric polarizability $\bar{\alpha}_{E}$ from real Compton scattering as discussed in [11,13]. The prediction of the $O\left(p^{3}\right)$ calculation for $\bar{\alpha}_{E}(0)=\bar{\alpha}_{E}$ agrees remarkably well with the experimental value extracted from real Compton scattering ( $\mathrm{HBChPT}\left[\mathrm{O}\left(p^{3}\right)\right]$ : $12.8 \times 10^{-4} \mathrm{fm}^{3}$; Expt.: $\left.(12.1 \pm 0.8 \pm 0.5) \times 10^{-4} \mathrm{fm}^{3}[2]\right)$. Slowly increasing three-momentum transfer $\bar{q}$, ChPT predicts a sharp decrease for the generalized electric polarizability $\alpha_{E}\left(\bar{q}^{2}\right)$ 
[17], which is considerably faster than predicted by the constituent quark model [11].

Likewise, we present the full $\bar{q}^{2}$ evolution of the generalized magnetic polarizability $\bar{\beta}_{M}\left(\bar{q}^{2}\right)$ in Fig.1]. At $\bar{q}^{2}=0$, it is falling short of the central experimental value from real Compton scattering $\left(\mathrm{HBChPT}\left[\mathrm{O}\left(p^{3}\right)\right]: 1.3 \times 10^{-4} \mathrm{fm}^{3}\right.$; Expt.: $(2.1 \mp 0.8 \mp 0.5) \times 10^{-4} \mathrm{fm}^{3}$ [2]). It is known that this discrepancy can be resolved in a $O\left(p^{4}\right)$ ChPT calculation, as at that order one can implement the effects of the nucleon resonance $\Delta(1232)$ [6]. In our $O\left(p^{3}\right)$ calculation, ChPT predicts $\bar{\beta}_{M}\left(\bar{q}^{2}\right)$ to rise at small momentum transfer, with a turnover point around $\bar{q}^{2}=0.1 \mathrm{GeV}^{2}$. Possible higher order modifications of this $\bar{q}^{2}$ behavior due to $\Delta(1232)$ are currently under investigation [20].

Finally, let us address the seven spin-dependent GPs, constrained by Eqs.(2b)-(2d). At this point, there exists not even a measurement of the corresponding polarizabilities in real Compton scattering to compare with. The possibility of such measurements is being studied [21]. We also note that the familiar "spin-polarizability" $\gamma$ of the nucleon [5, [6] is not accessible in the kinematical approximation of [11] as discussed in [14]. Our predictions for the spin-dependent GPs, shown in Fig.1, contain two rather distinct contributions - the first originating from pionic loop contributions, whereas the second is associated with the anomalous $\pi^{0} \gamma \gamma^{*}$ vertex. It is interesting that at $O\left(p^{3}\right)$ the contributions of the pion-nucleon loops to the spin-dependent GPs are much smaller than the contributions arising from the $\pi^{0}$-exchange diagram!

Experimentally, the extraction of the generalized (proton) polarizabilities is accomplished by utilizing the interference of the structure-dependent part of the VCS matrix element with the Bethe-Heitler and nucleon pole diagrams. Once $C$ invariance is imposed, in the kinematical approximation of Guichon et al. [11] the lowest-order structure-dependent contributions in an unpolarized experiment can then be parameterized in terms of three independent response functions [14], each containing products of nucleon form factors with the GPs as building blocks:

$P_{L L}\left(\bar{q}^{2}\right)=-2 \sqrt{6} M G_{E}\left(Q_{0}^{2}\right) P^{(01,01) 0}\left(\bar{q}^{2}\right)$, 


$$
\begin{aligned}
& P_{T T}\left(\bar{q}^{2}\right)=\frac{3}{2} G_{M}\left(Q_{0}^{2}\right)\left[2 \omega_{0} P^{(01,01) 1}\left(\bar{q}^{2}\right)+\sqrt{2} \bar{q}^{2}\left(P^{(01,12) 1}\left(\bar{q}^{2}\right)+\sqrt{3} \hat{P}^{(01,1) 1}\left(\bar{q}^{2}\right)\right)\right] \\
& P_{L T}\left(\bar{q}^{2}\right)=\sqrt{\frac{3}{2}} \frac{M \bar{q}}{\sqrt{Q_{0}^{2}}} G_{E}\left(Q_{0}^{2}\right) P^{(11,11) 0}\left(\bar{q}^{2}\right)+\frac{\sqrt{3} \sqrt{Q_{0}^{2}}}{2 \bar{q}} G_{M}\left(Q_{0}^{2}\right)\left(P^{(11,00) 1}\left(\bar{q}^{2}\right)+\frac{\bar{q}^{2}}{\sqrt{2}} P^{(11,02) 1}\left(\bar{q}^{2}\right)\right) .
\end{aligned}
$$

Here $G_{E}\left(Q^{2}\right)\left(G_{M}\left(Q^{2}\right)\right)$ are the electric (magnetic) nucleon Sachs form factors and $Q_{0}^{2}=$ $\left.Q^{2}\right|_{\omega^{\prime}=0}$. The ChPT predictions for the response functions are given in Fig. 2 . We note that the particular kinematic approximation suggested in [11] has the remarkable property that the $\pi^{0}$ pole contributions cancel exactly at the level of the response functions in an $O\left(p^{3}\right)$ calculation. Therefore, the information displayed in Fig.2 is directly based upon the pion-nucleon loop effects and the parameterization of the nucleon form factors. We have chosen to use a Taylor expansion of the nucleon form factors up to the first $Q^{2}$ coefficient in addition to the standard dipole-form. In Fig.1] one can see the differences between the parameterizations, which we take as a measure of the uncertainty of the $O\left(p^{3}\right)$ ChPT prediction. According to our analysis, the response function $P_{L L}\left(\bar{q}^{2}\right)$ is the one that can be predicted most reliably. It directly allows for extracting $\bar{\alpha}_{E}\left(\bar{q}^{2}\right)$.

The Mainz experiment (G. Audit et al. [8]) will supply data at $\bar{q}=600 \mathrm{MeV}$, which can be used to determine $\bar{\alpha}_{E}\left(\bar{q}^{2}=0.36 G e V^{2}\right)$ and two additional linear combinations of the GPs. The $O\left(p^{3}\right)$ ChPT calculation may not be valid at this "large" momentum transfer, but Fig.2 predicts that $\bar{\alpha}_{E}$ should have decreased by $\approx 50 \%$ from its real-photon value. It will be interesting to collect data at smaller $\bar{q}^{2}$, as it is planned in the $\bar{\alpha}_{E}$ optimized experiment at MIT-Bates ( J. Shaw et al. [8]). At this relatively small momentum transfer $(\bar{q} \approx 240 \mathrm{MeV})$ the $O\left(p^{3}\right)$ HBChPT calculation of the GPs should be most reliable. However, when $\bar{q}$ approaches the order of magnitude of $\omega^{\prime}$ the response function analysis of [11] will break down, due to the higher-order terms in $\omega^{\prime}$ which are neglected therein. For even smaller $\bar{q}$ one then approaches the kinematical regime that has been dealt with in [17. In summary the two above experiments taken together with the value of $\alpha_{E}(0)$ provide the first opportunity to determine the $\bar{q}^{2}$ dependence of a GP.

We have presented the first complete ChPT calculation for the GPs of the nucleon, 
which are going to be measured in several upcoming experiments. Future work will include an $O\left(p^{4}\right)$ calculation and the consideration of explicit $\Delta(1232)$ degrees of freedom. Work in these directions is in progress [20].

Research supported in part by NSERC, NSF, DAAD(HSPIII) and DFG(SFB201). 


\section{REFERENCES}

[1] F.E. Low, Phys. Rev. 96, 1428 (1954); M. Gell-Mann and M.L. Goldberger, Phys. Rev. 96, 1433 (1954).

[2] B.E. MacGibbon et al., Phys. Rev. C 52, 2097 (1995).

[3] B.R. Holstein, Comm. Nucl. Part. Phys. 20, 301 (1992).

[4] J. Gasser and H. Leutwyler, Ann. Phys. (N.Y.) 158, 142 (1984).

[5] E. Jenkins and A. Manohar, Phys. Lett. B 255, 558 (1991); V. Bernard, N. Kaiser, J. Kambor, and U.-G. Meißner, Nucl. Phys. B388, 315 (1992); V. Bernard, N. Kaiser, U.-G. Meißner, Int. J. Mod. Phys. E 4, 193 (1995).

[6] V. Bernard, N. Kaiser, U.-G. Meißner, and A. Schmidt, Phys. Lett. B 319, 269 (1993); Z. Phys. A 348, 317 (1994); T.R. Hemmert, B.R. Holstein, and J. Kambor, Phys. Rev. D 55, 5598 (1997).

[7] Proceedings of the Workshop on Virtual Compton Scattering VCS96, Clermont-Ferrand, June 1996, Ed: V. Breton.

[8] G. Audit et al., CEBAF Report No. PR 93-050, 1993; J. F. J. van den Brand et al., CEBAF Report No. PR 94-011, 1994; G. Audit et al., MAMI proposal "Nucleon structure study by Virtual Compton Scattering." 1995; J. Shaw et al., MIT-Bates proposal No. 97-03, 1997.

[9] R.A. Berg and C.N. Lindner, Nucl. Phys. 26, 259 (1961).

[10] H. Arenhövel and D. Drechsel, Nucl. Phys. A233, 153 (1974).

[11] P.A.M. Guichon, G.Q. Liu, and A.W. Thomas, Nucl. Phys. A591, 606 (1995); Aust. J. Phys. 49, 905 (1996).

[12] S. Scherer, A.Yu. Korchin, and J.H. Koch, Phys. Rev. C 54, 904 (1996). 
[13] D. Drechsel, G. Knöchlein, A. Metz, S. Scherer, Phys. Rev. C 55, 424 (1997).

[14] D. Drechsel, G. Knöchlein, A.Yu. Korchin, A. Metz, and S. Scherer, Report No. nuclth/9704064.

[15] A. Metz and D. Drechsel, Z. Phys. A 356, 351 (1996); Report No. nucl-th/9705010.

[16] M. Vanderhaeghen, Phys. Lett. B 368, 13 (1996).

[17] T.R. Hemmert, B.R. Holstein, G. Knöchlein, and S. Scherer, Phys. Rev. D. 55, 2630 (1997).

[18] M. Kim and D.-P. Min, Report No. hep-ph/9704381.

[19] J. Wess and B. Zumino, Phys. Lett. 37B, 95 (1971); E. Witten, Nucl. Phys. B223, 422 (1983).

[20] T.R. Hemmert, B.R. Holstein, and G. Knöchlein, in preparation.

[21] R. Miskimen, private communication. 


\section{FIGURES}

FIG. 1. $O\left(p^{3}\right)$ prediction for the GPs of the proton as a function of $\bar{q}^{2}$. The dashed line represents the contribution from pion-nucleon loops, the dotted one from the $\pi^{0}$ exchange graph and the dash-dotted line the sum of both.

FIG. 2. Response functions in $e p \rightarrow e^{\prime} p^{\prime} \gamma$. The dashed line results from a dipole-parameterization of the proton form factors with $G_{D}\left(Q^{2}\right)=\left[1+Q^{2} /\left(0.71 \mathrm{GeV}^{2}\right)\right]^{-2}$, whereas the dotted curve utilizes the Taylor expanded formfactors $G_{T}\left(Q^{2}\right)=1+\left.Q^{2} \frac{d G_{D}}{d Q^{2}}\right|_{Q^{2}=0}$. The difference between the two curves represents a measure for the importance of $O\left(p^{n}\right), n>3$ corrections. 

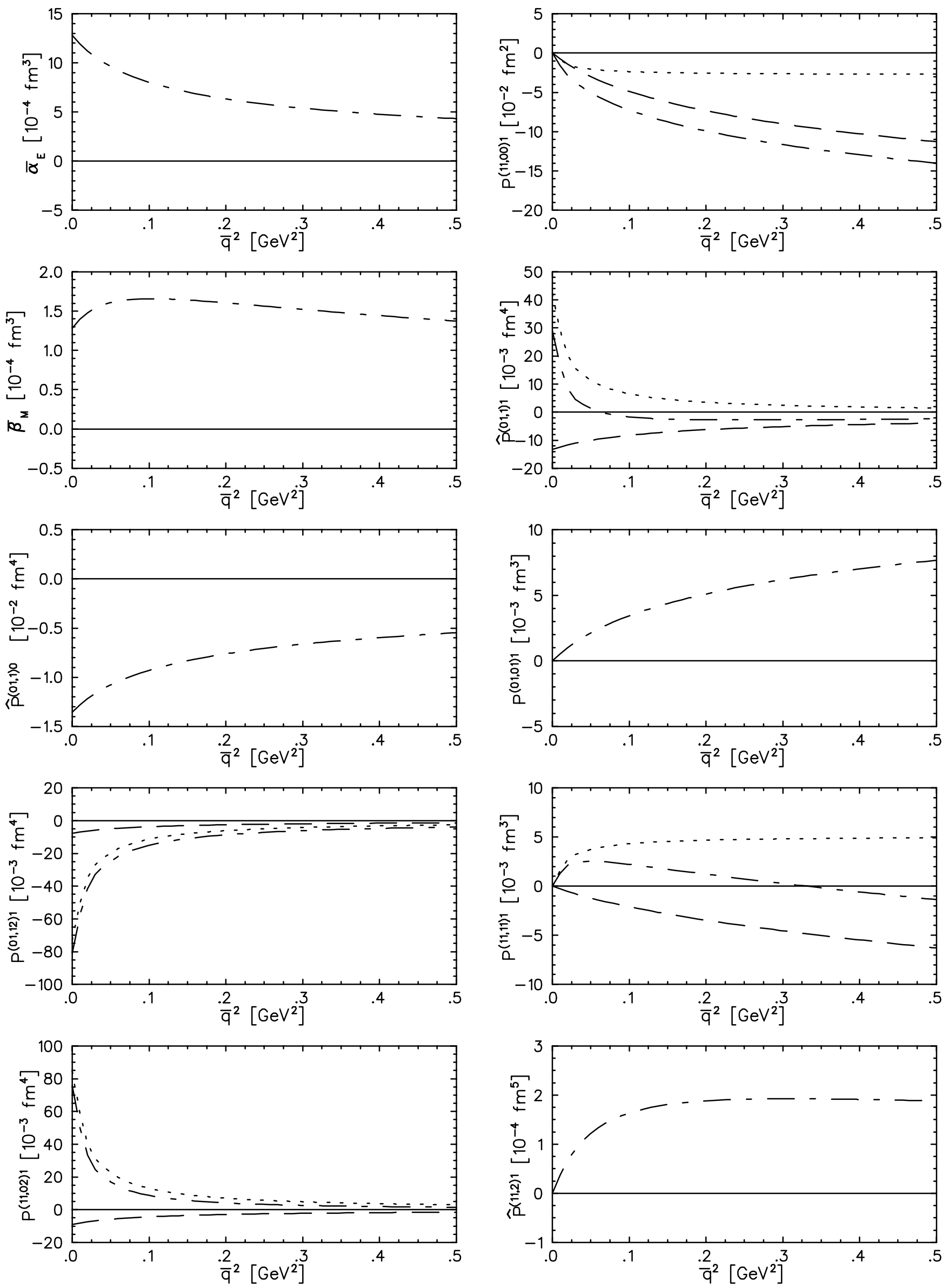

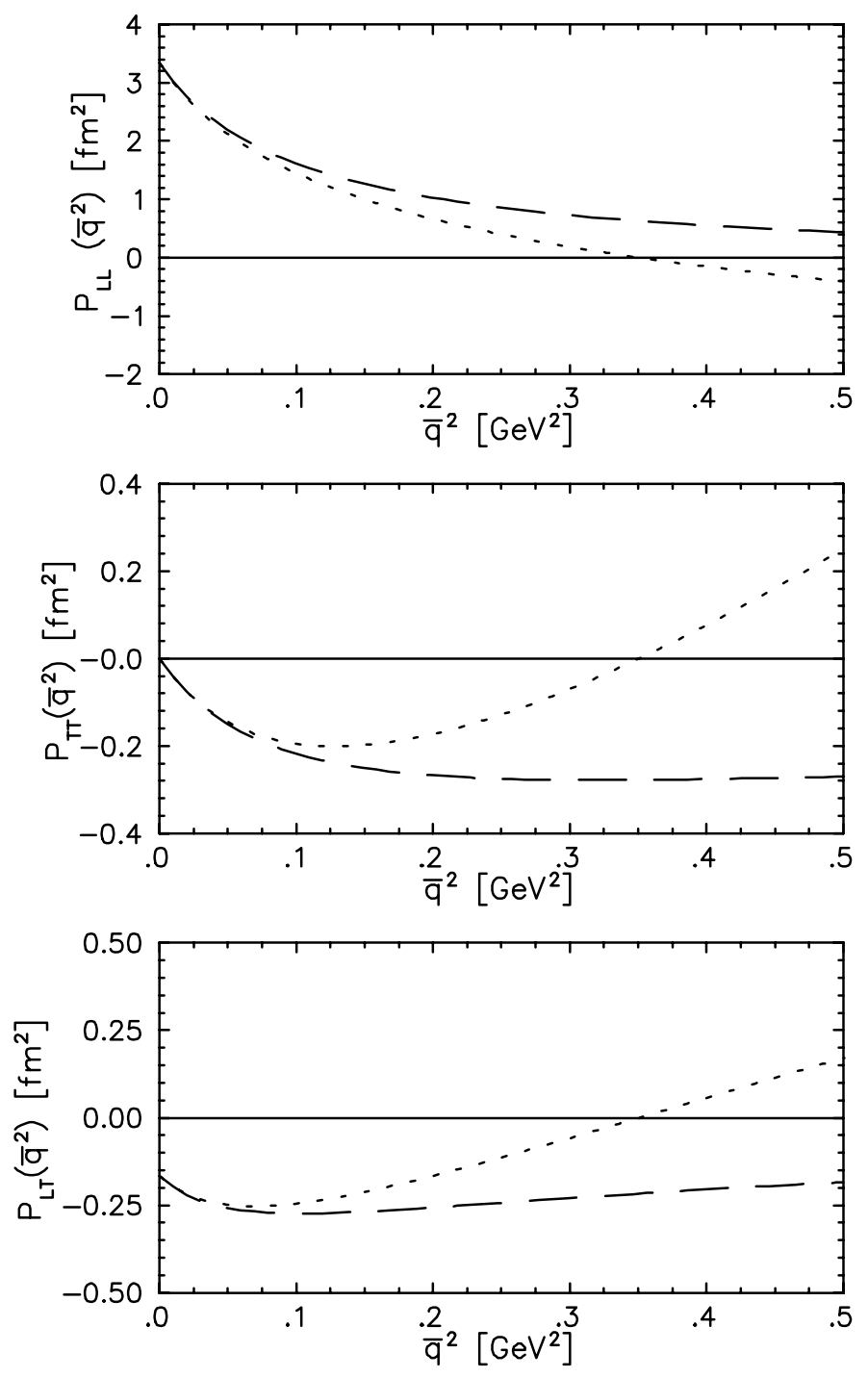